\title{
NPC, A NEW COMBINED POSITION CATALOGUE OF STARS IN THE NORTHERN POLE REGION
}

\author{
L. G. TAFF \\ Department of Physics and Astronomy, J. Hopkins University \\ Baltimore, Maryland, USA \\ AND \\ V.V. TEL'NYUK-ADAMCHUK, O.A. MOLOTAJ \\ Astronomical Observatory, Taras Shevchenko University \\ Kyiv, Ukraine
}

\begin{abstract}
The main task of this work was to construct a new catalogue of positions and proper motions of stars in the north polar cap, and using the results obtained to study the systematic features of the source catalogues as well as the reference system in the polar region. To achieve the goal, thirteen source catalogues, both meridian and photographic, covering an epoch span near one and a half century, were collected. Most of them were not involved in the process of both the PPM and the ACRS constructions. The new combined catalogue of positions and proper motions, named North Polar Catalogue (NPC), lists 4272 stars on the FK5 system, J2000.0. The catalogue was constructed using the method of infinitely overlapping circles. It represents the second use of this procedure to construct a star catalogue. External rms accuracy of positions for the epoch of 2000 is equal to $0^{\prime \prime} 15$ and $0 \prime 25$ for the centennial proper motions, respectively. The internal rms errors of positions at the mean catalogue epoch, near 1940, are equal to 0 !' 18 and $0^{\prime \prime} 15$ for right ascension and declination correspondingly, while external rms position errors for that epoch are of the order of $0^{\prime \prime} 05$. Comparisons were made between the NPC and three modern catalogues, the FK5, PPM, and ACRS. The systematic differences between the new catalogue and PPM one for the epoch of 2000 are valuable, especially in the case of errors depending on right ascension. The authors consider as an essential part of the systematic differences that, which is caused by some shortcomings of the PPM in the polar zone. For the mean epoch of the new catalogue positions the systematic differences are smaller.
\end{abstract}

I. M. Wytrzyszczak, J. H. Lieske and R.A. Feldman (eds.), Dynamics and Astrometry of Natural and Artificial Celestial Bodies, 499, 1997.

(C) 1997 Kluwer Academic Publishers. Printed in the Netherlands. 
This paper gives a result of the first part of investigation of near-polar regions, both the northern and southern. The principal aims of it are as follows: (1) to collect available catalogues of near-polar stars, including both the rarely utilized catalogues, and the modern ones; (2) to investigate in unified approach each catalogue using the comparison with the modern observations, as well as combined and fundamental catalogues; (3) to estimate their random and systematic accuracy and to choose the suitable catalogues as to our interest; (4) to use the conventional rectangular coordinate system to avoid specific problems which arise due to the equatorial system singularity; to use the appropriate catalogue intercomparison method which should be effectively working in the polar region; (5) to compile a combined catalogue of positions and proper motions of near-polar stars above a declination of $+80^{\circ}$ using a wide observational base; (6) to make the resulting system on the FK5, J2000.0; and (7) to use a catalogue constructed to study the systematic features of modern catalogues in the zone under consideration.

Thirteen source catalogues, eight meridian and five astrographic, have been collected (see Table 1). Most of them have not been utilized when constructing as the PPM catalogue by Roeser and Bastian (1991), as the ACRS one by Corbin and Urban (1991).

As a standard, the FK5 system has been chosen. We used three FK5 representatives: FK5 Basic by Fricke et al. (1988), FK5 Extension by Fricke et al. (1991), and the improved positions and proper motions of 995 stars by Schwan et al. (1993). Two stars from the FK5 Extension were omitted, i.e. BD $83^{\circ} 365$ and $85^{\circ} .222$, because of their obviously mistaken positions. This subset of stars compiled in such a way we refer to FK5*. To improve the tie between the source catalogues and the fundamental system, the FK5* stars were used down to $75^{\circ}$ declination. Beginning from early stages of our work we dealt with the J2000.0 equinox. The source catalogues have been transformed to this equinox according to Smith et al. (1989). If needed, the computations were carried out considering each coordinate separately when the observation epoch of $\alpha$ and $\delta$ differed. To estimate the quality of the source catalogues, fields were built of the systematic differences by averaging the individual differences between the PPM catalogue and contributed ones. It might be noted, that the more remote a catalogue observation epoch is, the larger the differences. We can explain that by the features of the PPM catalogue proper motions.

While dealing with heterogeneous observational data we really are faced with the problem of how to combine the catalogues, both meridian and astrographic, having so different epochs of observations and produced in several different ways. Lack of clear ways to resolve the problem within the framework of available data leads us to use the magnitude and color system of the PPM catalogue, as representative of the FK5 system with the same 
TABLE 1. Source catalogues.

\begin{tabular}{|c|c|c|c|c|c|c|c|}
\hline No & Catalogue & $\begin{array}{l}\text { Number } \\
\text { of stars }\end{array}$ & $\begin{array}{l}\text { Mean } \\
\text { epoch }\end{array}$ & Equinox & System & Dsc & $\mathrm{Rms}$ \\
\hline 1 & $\begin{array}{l}\text { Carrington } \\
\text { (Carrington, 1857) }\end{array}$ & 3735 & 1855 & 1855.0 & $\begin{array}{l}\text { Car- } \\
\text { rington }\end{array}$ & 0.82 & $\begin{array}{l}0.70 \\
0.60\end{array}$ \\
\hline 2 & $\begin{array}{l}\text { Fabritius } \\
\text { (Tel'nyuk-Adamchuk and } \\
\text { Molotaj, 1992) }\end{array}$ & 550 & 1880 & B1950.0 & FK4 & 0.42 & $\begin{array}{l}0.30 \\
0.30\end{array}$ \\
\hline 3 & $\begin{array}{l}\text { Greenwich meridian } \\
\text { (Greenwich, 1909) }\end{array}$ & 1501 & 1901 & 1900.0 & $\begin{array}{l}\text { Green- } \\
\text { wich }\end{array}$ & 0.39 & $\begin{array}{l}0.30 \\
0.30\end{array}$ \\
\hline 4 & $\begin{array}{l}\text { Greenwich astrographic } \\
\text { (Astrographic catalogue, } \\
\text { 1914) }\end{array}$ & 4502 & 1901 & 1900.0 & $\begin{array}{l}\text { Green- } \\
\text { wich }\end{array}$ & 0.28 & $\begin{array}{l}0.20 \\
0.20\end{array}$ \\
\hline 5 & $\begin{array}{l}\text { Berlin } \\
\text { (Freundlich, 1916) }\end{array}$ & 1484 & 1912 & 1910.0 & $\begin{array}{l}\text { Pola- } \\
\text { rissima }\end{array}$ & 0.56 & $\begin{array}{l}0.30 \\
0.40\end{array}$ \\
\hline 6 & $\begin{array}{l}\text { Kharkiv-1, Decl. only } \\
\text { (Kuz'menko et al., 1982) }\end{array}$ & 1294 & 1912 & B1950.0 & FK4 & 0.36 & $\overline{0}-\overline{0}$ \\
\hline 7 & $\begin{array}{l}\text { Pulkovo } \\
\text { (Belyavsky, 1947) }\end{array}$ & 4155 & 1930 & B1950.0 & $?$ & 0.31 & $\begin{array}{l}0.15 \\
0.15\end{array}$ \\
\hline 8 & $\begin{array}{l}\text { AGK2 } \\
\text { (Dieckvoss et al., 1975) }\end{array}$ & 4317 & 1930 & B1950.0 & FK4 & 0.12 & $\begin{array}{l}0.25 \\
0.30\end{array}$ \\
\hline 9 & $\begin{array}{l}\text { Yale (Barney and } \\
\text { van Woerkom, 1954) }\end{array}$ & 1031 & 1951 & B1950.0 & USNO & 0.08 & $\begin{array}{l}0.10 \\
0.10\end{array}$ \\
\hline 10 & $\begin{array}{l}\text { AGK3 } \\
\text { (Dieckvoss et al., 1975) }\end{array}$ & 4317 & 1960 & B1950.0 & FK4 & 0.12 & $\begin{array}{l}0.15 \\
0.15\end{array}$ \\
\hline 11 & $\begin{array}{l}\text { Kharkiv-3, R.A. only } \\
\text { (Derkach, 1994) }\end{array}$ & 452 & 1964 & B1950.0 & FK4 & 0.35 & $\begin{array}{l}0.20 \\
-\end{array}$ \\
\hline 12 & $\begin{array}{l}\text { Kharkiv-2, R.A. only } \\
\text { (Vantzan, 1993) }\end{array}$ & 550 & 1984 & B1950.0 & FK4 & 0.22 & $\begin{array}{l}0.15 \\
-\end{array}$ \\
\hline 13 & $\begin{array}{l}\text { CAMC (Carlsberg, 1989, } \\
1991,1992,1993,1994)\end{array}$ & 1462 & 1990 & $\mathrm{~J} 2000.0$ & FK5 & 0.18 & $\begin{array}{l}\text { Ori- } \\
\text { ginal }\end{array}$ \\
\hline
\end{tabular}

Remark to Table 1: Dsc - random rms differences between positions in the PPM catalogue and source catalogue for the mean epoch of observations; Rms - right ascension (above) and declination (below) rms accuracy in arcsec adopted for catalogue positions while determining the positions and proper motions of the combined catalogue.

star density. Removing step by step the systematic components "PPM minus Source Catalogue" depending upon $\alpha, \delta$, magnitude, and spectral class from individual differences we treated the two last components as corrections to star positions in the individual catalogues to bring them onto the PPM magnitude and color system which can be treated as close to the FK5 system. Thus, the system of resulted combined catalogue with respect to 
magnitude and color is borrowed from the PPM catalogue. For the sake of the final bringing to the FK5 system and homogenization of individual catalogues we used the filter determined by the method of infinitely overlapping circles (Taff et al., 1990). As a result the Combined Catalogue of Positions and Proper Motions of 4272 Near-Polar Stars North of $80^{\circ}$ Declination was obtained (afterwards referred to as North Polar Catalogue, NPC). A quick statistical summary of the NPC catalogue's properties follows: total number of star positions used $-28,728$; total number of stars 4,272 ; average number of catalogue places per star - 6.6; weighted epoch for $\alpha$ and $\delta-1942$ and 1937, respectively; the total internal random rms errors: in $\alpha$ at the mean epoch of $\alpha-0.08$, in $\delta$ at the mean epoch of $\delta-0 . \prime 08$, that for the epoch of $2000-0.21$ and 0.23 , respectively, in $\mu_{\alpha}$ and $\mu_{\delta}-0.32^{\prime \prime} / \mathrm{cy}$ and 0.33 "/cy, respectively; the total external random rms error as a result of the NPC comparison with the PPM and ACRS catalogues: in $\alpha$ for the epoch of $2000-0$ ! 18 , that for the mean epoch 0 " 05 , in $\delta$ for the epoch of $2000-0$ " 15 , that for the mean epoch - 0 " 04 , in $\mu_{\alpha}-0.25^{\prime \prime} / \mathrm{cy}$, in $\mu_{\delta}-0.22^{\prime \prime} / \mathrm{cy}$.

To study the external accuracy the NPC was compared with the FK5*, PPM, and ACRS (part 1) catalogues. The position differences are too small especially for the mean epoch (see Table 2). Differences in $\delta$ are essentially larger as compared to that in $\alpha$. The variations of the differences in the proper motions illustrate a strong similarity with those of the positions. Thus, we conclude that position differences are caused by differences in the proper motion system of catalogues. Table 2 illustrates also the significant shift of PPM positions as compared with the ACRS and NPC.

TABLE 2. Systematic shift, in arcsec, in the positions and centennial proper motions between the PPM, ACRS, FK5*, and NPC catalogues in the near-polar zone at the epoch of 2000 and at the mean epoch of NPC (near 1940, in brackets).

\begin{tabular}{lllllll}
\hline Differences & $\Delta \alpha$ & $\Delta \delta$ & $\Delta \mu_{\alpha}$ & $\Delta \mu_{\delta}$ & $N_{1}$ & $N_{2}$ \\
\hline PPM - NPC & $+0.0^{\prime \prime}(-0 . \prime 03)$ & $-0.10(+0.10)$ & +0.21 & -0.32 & 4174 & 59 \\
ACRS - NPC & $-0.05(0.00)$ & $+0.01(-0.01)$ & -0.08 & +0.04 & 1868 & 24 \\
FK5* - NPC & $+0.01(0.00)$ & $-0.00(0.00)$ & +0.01 & -0.01 & 114 & 0 \\
PPM - ACRS & $+0.10(-0.02)$ & $-0.13(+0.10)$ & +0.20 & -0.36 & 2300 & 45 \\
\hline
\end{tabular}

Remark to Table 2: $N_{1}$ denotes the number of individual differences used to form averaged values; $N_{2}$ is the number of stars whose position differences exceed 1.5 arcsec and are ignored, $\left(N_{1}+N_{2}\right)$ is a number of common stars in these catalogues.

Figure 1 shows the maps of the PPM-NPC, ACRS-NPC, PPM-ACRS, and $\mathrm{FK} 5^{*}-\mathrm{NPC}$ differences, averaged within the circles of one-degree radius. Because of deficit of stars in the case of the $\mathrm{FK} 5^{*}-\mathrm{NPC}$ maps, we 

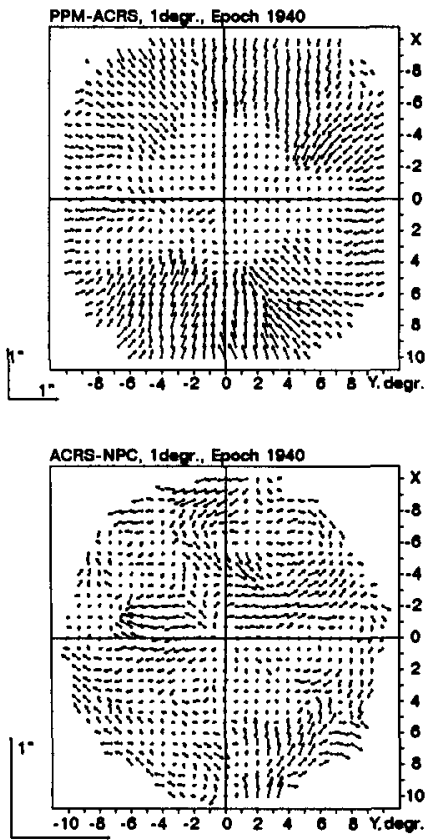
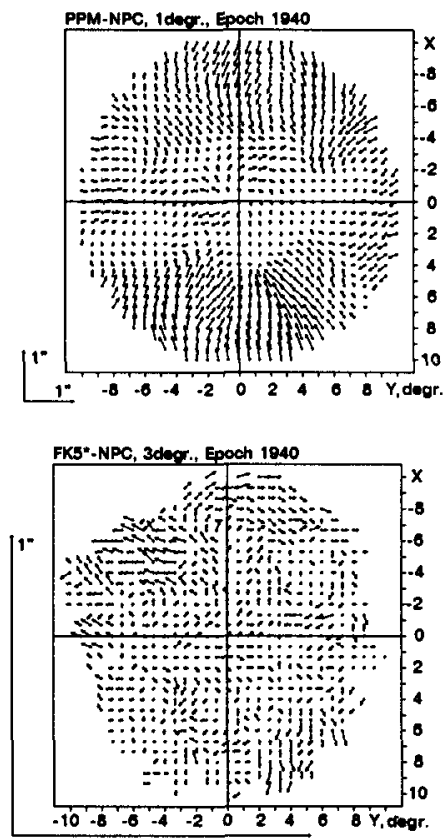

Figure 1. The maps of systematic differences PPM-ACRS, PPM-NPC, ACRS-NPC, and FK5*-NPC in rectangular coordinates (as seen from the North Pole) for the NPC mean epoch. Scales of one arcsec are shown in the bottom left corner of each map.

used three-degree radius. A moving average filter was applied by computing the mean differences in the circles centered in uniformly distributed points. Very similar pictures as well as some central symmetry of maps display the PPM-ACRS and PPM-NPC differences either for the epoch 2000 or for the mean epoch of the NPC. At the epoch of 1940, differences are substantially smaller, symmetry is clearly visible. Moreover, differences are clearly visible between the PPM near-polar stars and stars south of $85^{\circ}$ declination. As compared to the PPM-ACRS/NPC the FK5*-NPC differences are quite small, especially at the mean epoch of NPC where they are negligible. Individual differences NPC-PPM, plotted against $\alpha$, show very interesting peculiarities. The near-two-hour periodicity in the $\Delta \alpha_{\alpha}$ is clearly seen in $0-8^{h}$ range for 2000 epoch. $\Delta \delta_{\alpha}$, calculated for the NPC mean epoch, illustrates a clearly visible splitting of the differences into two distinguished subsets above and below $85^{\circ}$ declination. Such peculiarities are not seen in the patterns of the ACRS - NPC differences, while they are visible in the PPM-ACRS ones. So, one can link these peculiarities with the PPM catalogue. Moreover, we can deduce that AGK data are responsible of such a periodicity.

The NPC is now available in machine-readable form and can be obtained by sending an e-mail message to vtel@aoku.freenet.kiev.ua. 
Acknowledgements. This work is supported in part by NASA grant NAGW2597, by the American Astronomical Society, and by grant 2.3/666 of the Fundamental Research State Fund of Ukraine.

\section{References}

Astrographic catalogue 1900.0. Greenwich section 3, 1914, Edinburgh.

Belyavsky, S.I.: 1947, "Astrographic catalogue of 11332 stars between 70 degree of north declination and north pole", Trudy Glavnoy Astron. Observ. v Pulkove 60, 1.

Barney, I. and van Woerkom, A.J.J.: 1954, "Catalogue of the positions and proper motions of 1031 stars", Trans. Astron. Obs. Yale Univ. 26, 1.

Carlsberg Meridian Catalogue La Palma, 1989 4; 1991 5; 1992 6; 1993 7; 19948.

Carrington, R.G.: 1857, A Catalogue of 3735 Circumpolar Stars Observed at Redhill in Year 1854, 1855, and 1856, and Reduced to Mean Positions for 1855.0., London.

Corbin,T.E. and Urban, S.E.: 1991, "Astrographic catalogue reference stars", Doc. No. NSSDC/ WDC-A-R\&S 91-10.

Derkach, K.N.: 1994, "Differential catalogue of right ascension of 476 faint near-pole stars on epoch of observation and B1950.0 equinox received using observations with Kharkiv meridian circle during $1958-1965$ ", private communication.

Dieckvoss, W., et al.: 1975, AGK3. Star Catalogue of Positions and Proper Motions North of $-2.5^{\circ}$ Declination 1.

Freundlich, E.: 1916, "Katalog von 1886 Sternen zwischen +79 und $+90^{\circ}$ Deklination", Veröff. der Königlichen Sternwarte zu Berlin-Babelsberg 2, h.1.

Fricke, W., et al.: 1988, "Fifth fundamental catalogue, Part I. The basic fundamental stars", Ver̈off. Astron. Rechen-Inst. Heidelb., No 32.

Fricke, W., et al.: 1991, "Fifth fundamental catalogue, Part II. The FK5 extension - new fundamental stars", Veröff. Astron. Rechen-Inst. Heidelb., No 33.

Greenwich Second Nine-Year Catalogue of Stars for 1900.0, 1909, Edinburgh.

Kuz'menko, K.N., Kyrpatovsky, V.M., and Pavlenko, L.S.: 1982, "Kharkiv differential catalogue of declinations of 1407 near-pole stars on the FK4 system for epoch of 1911", manuscript deposited in VINITI, Moscow, 1982 Jun 24, No 3641-82 Dep.

Roeser, S. and Bastian, U.: 1991, PPM Star Catalogue 1, Heidelberg, Spectrum Acad. Verlag.

Schwan, H., et al.: 1993, "Improved mean positions and proper motions for the 995 FK4 sup stars not included in the FK5 extension", Veröff. Astron. Rechen-Inst. Heidelb., No 34 .

Smith, C.A., et al: 1989, Astron. J. 97, 265.

Taff, L.G., Bucciarelli, B., and Lattanzi, M.G.: 1990, Astrophys. J. 361, 667.

Tel'nyuk-Adamchuk, V.V. and Molotaj, A.A.: 1992, "Catalogue of positions and proper motions of 520 near-pole stars on the FK5 system for equinox and epoch of J2000.0", manuscript deposited in UkrINTEI, Kyiv, 1992 Apr 28, No 494 -Uk92.

Vantsan, A.F.: 1993, "Differential catalogue of right ascension of 630 near-pole stars, received using meridian observations in Kharkiv during 1981-1984", manuscript deposited in UkrINTEI, Kyiv, 1993 March 12, No 477 -Uk93. 